\title{
Reproductive tract microbiota of women in childbearing age shifts upon type of gynecological infections and phase in physiological cycle
}

\section{Yan Gao}

Zhangdian District Center for Disease Control and Prevention

Hongyan Liu

Institute of Basic Medicine,Shandong First Medical University \& Shandong Academy of Medical

Sciences

\section{Lijuan Cheng}

The Second Affiliated Hospital,Shandong University of Traditional Chinese Medicine

\section{Qing Xia}

Shandong University Qilu Hospital

\section{Xiuzhen Xie}

The Second Affiliated Hospital,Shandong University of Traditional Chinese Medicine

\section{Hui Wang}

The Second Affiliated Hospital,Shandong University of Traditional Chinese Medicine

\section{Heying Shang}

The Second Affiliated Hospital,Shandong University of Traditional Chinese Medicine

\section{Yurong Liu}

Obstetrics and Gynecology Department,The 5th People's Hospital of Jinan

\section{Yutao Diao ( $\nabla$ dyutium@sina.com )}

Institute of Basic Medicine,Shandong First Medical University \& Shandong Academy of Medical

Sciences

\section{Research article}

Keywords: Microbiome, Microbiota, Lactobacillus, alpha diversity, beta diversity, relative anundance

Posted Date: June 29th, 2020

DOI: https://doi.org/10.21203/rs.3.rs-37456/v1

License: (c) (1) This work is licensed under a Creative Commons Attribution 4.0 International License. Read Full License 


\section{Abstract}

Background: The association of the normal physiological cycle to the structural pattern of microbiota in reproductive tract of women at reproductive age has not been extensively explored. This study was undertaken to determine whether the vaginal microbes of women at childbearing age is different among groups defined by urogenital tract infections, childbearing history and menstrual cycle, respectively.

Results: This was a multiple case-control study of women at childbearing age who were assigned to case or control groups according to their states of urogenital tract infections. The participants were also grouped by childbearing history and menstrual cycle. Samples of vaginal swabs were collected and stored at $-70^{\circ} \mathrm{C}$ until assayed. The V3-V4 regions of $16 \mathrm{~S}$ rRNA genes were amplified using PCR and sequenced on the Illumina MiSeq platform. We tested the hypothesis of whether the relative abundance of microbial species in vaginal microbiota was different between women with different urogenital tract infections, childbearing history and menstrual cycle. We showed that the vaginal microbial richness(Alpha diversity measured by PD_whole tree) was decreased in normal women(without reproductive tract infections) than in those with bacterial vaginosis (BV), and decreased in pregnant women than in other groups of non-pregnancy. Similarly, women from groups of normal and in pregnancy had lower beta diversity on measure of unweighted_unifrac distance in comparison to those of uninfected and non-pregnant. The top 10 genus relative abundance, especially that Lactobacillus was the most dominant genus with the relative abundance of $71.55 \%$ among all samples, did not differ significantly between groups of childbearing history and menstrual cycle analyzed by ANOVA and nonparametric kruskal_wallis.

Lactobacillus iners and Lactobacillus helveticus have the most abundance, totally account for $97.92 \%$ relative abundance of genus Lactobacillus. It is proposed that a higher L.helveticus/ L.iners ratio is more likely to present in normal women than in the infected and in pregnant than in non-pregnant, although this comparison lacks statistical significance.

Conclusions: The relative abundance of dominant bacterial taxa in vaginal microbial communities of women at childbearing age, characterized with 16S rRNA gene sequence and QIIME based analysis, were not different among groups of childbearing history and menstrual cycle. Women from groups of in pregnancy and without reproductive tract infections had lower alpha and beta diversity. The compositional ratio of the main lactobacillus species may shift depending on the normal physiological cycle and reproductive tract infections.

\section{Background}

Woman genital tract is one of the natural niches that harbor the most amount of body microbes[1].The normal structure of vaginal microbiome plays a pivotal role in maintaining the healthy microecosystem, especially for those in childbearing age. Any deviation of this eubiosis can lead to disorders such as bacterial vaginosis(BV), mycotic vaginitis(MV) or pelvic inflammation, which significantly impact the 
health of women, their fetuses and new born infants[2-4]. Furthermore, the role of dysbiosis in causing gynecological cancers has been appreciated only recently[5]. On the other hand, the composition of genital tract microbiota differs depending on factors that have no direct relation to infection, such as race, nationality that the women belonging to[6], or hygiene practices that they accustomed to[7,8]. Quite few reports dealt with the impact of a natural menstrual cycle on bacterial growth, colonization, and community structure. The others were about the comparison of vaginal microbial composition of nonpregnant with pregnant women by the case-control longitudinal study but these designs did not reflect on the real menstrual cycle[9,10]. We thus postulate that gynecological infections as well as the normal physiological cycle may affect the structural profile of vaginal microbiota of women in childbearing age. So the current study sought to examine this question by characterizing the differential microbiota among groups of urogenital tract infections, childbearing history and phases of menstrual cycle.

\section{Results}

The grouping and characteristics of the 111 study subjects were shown in Additional file:Table S1 and Fig S1. No differences in age_at_first_marriage, days of menstrual_cycle, and pH_of_vaginal_discharge were found by childbearing history, menstrual cycle, gynecological infections, respectively. The mean age of women without childbearing was significantly lower than that of women with childbearing and women in pregnancy.

The alpha diversity on measure of PD_whole tree other than chao1, observed_outs and Shanonn's index was lower in group of women in pregnancy compared to those with_childbearing $(P=0.021)$ and those in follicular phase $(P=0.048)$. Of the 5 groups on gynecological diagnosis, normal women had lower alpha diversity on measure of PD_whole tree in contrast to the other infected groups, but only the difference to BV group was statistically significant $(P=0.04)$ (Fig 1a-c, Additional file: Table S2). As for the measurement of beta diversity, normal women were clustered together based on their genital tract microbial taxa having closer genetic affinity(having shorter unweighted_unifrac distance) apart from those in women of $\mathrm{BV}$, mycotic vaginitis(MV) and $\mathrm{MV}+\mathrm{BV}$ group(Bonferroni-corrected parametric $P \otimes 0.001$, Bonferroni-corrected nonparametric $P=0.136$ ) (Fig 2a, Additional file: Table S3). With respect to grouping by childbearing history and menstrual cycle, women in pregnancy had conspicuous constitutional similarity of microbial population compared to those with or without childbearing group(Bonferronicorrected parametric $P=0.001$, Bonferroni-corrected nonparametric $P=0.028$.) (Fig $2 b$, Additional file: Table S3) Similarly, taxa in genital tract of pregnant women were significantly alienated from taxa in women that were in luteal_phase or follicular_phase(Bonferroni-corrected parametric $P \otimes 0.001$, Bonferronicorrected nonparametric $P=0.028)$. (Fig 2c, Additional file: Table S3).

The top 10 genus relative abundance among different groups did not differ significantly compared by ANOVA and nonparametric kruskal_wallis, with one exception that groups defined by gynecological infections had different relative abundance of 3 genera out of the top 10 genera tested by ANOVA(Fig 1d, Fig.3, Additional file: Table S4). The detail of these differences were intensively analysed by Bonferroni multiple comparisons. That is, group of mycotic vaginitis(MV) had higher relative abundance of genus 
Gardnerella compared to group of normal $(P=0.041)$ and had higher relative abundance of genus NA(affiliated to Lactobacillales orders) compared to group of normal $(P=0.042)$ and group of $\mathrm{BV}(P=0.025)$ (Fig 1d, Additional file: Table S5). Women affected nongonococcal urethritis/cervicitis(NUC) had higher relative abundance of Streptococcus in contrast to the others $(P<0.001)$ (Additional file: Table S5). The graph of NUC group was not included in Figure $1 \mathrm{~d}$ due to its $95 \%$ interval line disproportionately expanded caused by the low sample size(only 2 women). Lactobacillus is the most dominant over other taxa for sustaining normal vaginal ecosystem. As shown in figure $1 \mathrm{~d}$ and figure 3, Lactobacillus was the most dominant genus among all sample groups with the mean relative abundance of Lactobacillus being the highest in Normal group over other infection groups. But there were no significant relative abundance discrepancies between groups of gynecological infection in genus Lactobacillus, nor were in Atopobium and Prevotella, even though which had been classified as bacterial vaginosis associated bacteria(BVAB) or as markers for the other forms of urogenital tract infections(data not shown).

In addition to the common sense that Lactobacillus is the dominant population in normal (without infectious diseases) group of women, we found women in gestation also had a slight higher Lactobacillus relative abundance in comparison to women without pregnancy. Furthermore, women in gestation and without genital tract infections (in_pregnancy+normal) harbored the highest proportion of genus Lactobacillus over the other group of women(Fig 3). In order to scrutinize the exquisite constituent structure of genus Lactobacillus, we explored the compositional profile of all the 11 Lactobacillus species wherein Lactobacillus iners and Lactobacillushelveticus have the most abundance, totally account for 97.92\% relative abundance of genus Lactobacillus. It is proposed that a higher helveticus/ iners ratio is more likely to present in normal women than in the infected and in pregnant than in non pregnant, although this comparison lacks statistical significance(at 0.05 significant level)(Fig 4).

\section{Discussion}

We established that the structural pattern of vaginal microbiota characterized by high relative abundance of species of Atopobium as well as the presence of Prevotella, Sneathia, Gardnerella, Ruminococcaceae, Parvimonas, Mobiluncus and other taxa previously shown to be bacterial vaginosis associated bacteria(BVAB) was rarely observed in either pregnant or other groups of women. Nevertheless, it is found in all the samples that only Gardnerella and Streptococcus were significant indicator genus for mycotic vaginitis(MV) and nongonococcal urethritis/cervicitis(NUC), respectively. Although there were no significant relative abundance differences of the top 10 bacterial genus, which totally account for $96.25 \%$ microbe amount of the whole community, between pregnant and non-pregnant women, we observed the decreased microbial richness measured by PD_whole tree alpha diversity in pregnant than in nonpregnant women and in normal than in infected women. This result are contrary to recently reported by Yulian Chen,et al[11] in that they found both pregnancy and HPV infection can increase vaginal bacterial microbial richness and diversity. Although we consider the reason is they measured the microbial richness by Chao and Shannon index other than we used PD_whole tree that focus on the phylogenic affinity between each microbe, there must exist underlying factors that shield the real association of taxonomic diversity to pregnancy. Some intensive studies involving metagenome or 16S RNA gene 
sequencing are needed to identify the genomic function differences underlying the taxa diversity differences between microbiota in the corresponding groups defined by urogenital infections or normal physiological cycle[12-14].

Lactobacillus was the most dominant genus with the relative abundance of $71.55 \%$ among all 111 samples in this study. However, we did not find the presence of Lactobacillus vaginalis, L. crispatus, $L$.gasseri and $L$. jensenii at species level as reported in previous studies[15-17]. Indeed, the dominant members of Lactobacillus species has been limited and elusive based on the context of each study. In most cases, the vaginal microbiota through a menstrual cycle demonstrate that L.crispatus, L.iners, and L.jensenii was the dominant members[9]. Lactobacillus gasseri was considered prominent in other studies[15,16], but it was not a dominant organism in any woman's microbiota profile other than L.iners, and L. helveticus in this study, nor were L.crispatus, L. vaginalis, and L.jensenii.

There has been recent species-specific attention to L.iners which is commonly found in the vagina[15] and has been associated with both BV and healthy states[18-21]. In addition, L.iners is often the first Lactobacillus species to recover after treatment for BV[21,22]. But the role of L.iners in the dynamic balance between the family members of vaginal microbiota is still controversial. Some reports suggested there are strains of L.iners which are highly stable over time while others are associated with a rapidly changing vaginal microbiota toward a BV state[16,22]. Jakobsson et al[23] reported that $L$ iners is a dominant part of the vaginal flora when the flora is in a transitional stage from normal to abnormal while others observed that L.crispatus produce more $\mathrm{H}_{2} \mathrm{O}_{2}$ and bactericidal agents compared with other Lactobacilli species, so that it is considered the leading role in maintaining the health of vaginal ecosystem[24]. Unfortunately, we did not found L.crispatus in the specimens in the present and our previous study samples[25]. According to our findings, L.iners and L.helveticus totally account for $97.92 \%$ relative abundance of genus Lactobacillus with L.helveticus was discovered for the first time in this study as a leading member in genus Lactobacillus. Moreover, the increased proportion of L.helveticus over L.iners in normal and pregnant women than in other group of women indicates it may be the indicator for healthy status of reproductive tract microecology. We thus conjecture that women with L.iners-dominant and L.crispatus-absent intravaginal microbial flora, who is presumed to be normal by the phenotype methods, is thought to be at risk of BV or other urogenital tract infections and must be monitored carefully. Finished and ongoing work is needed to evaluate the genomic heterogeneity of L.iners and if different strains are associated with health and abnormal outcomes[26].

In brief, the current study engaged on multiple designs to explore the compositional differences of vaginal microbes between groups of women at reproductive age defined by urogenital tract infections, childbearing history and menstrual cycle, respectively. The vast volume work of sequencing data processing and analysis was performed on QIIME2 and QIIME 1.9.0 platform embedded in Linux operational system in addition to some outcomes resulted from QIIME was analyzed by SPSS 22.0. These methods should have identified significant differences among groups of women defined by urogenital tract infections, childbearing history and menstrual cycle. Weaknesses include the modest sample size, especially for NUC sample group that had only 2 women, which contributed to the larger 
variance in the comparison of outcomes between groups using parametric methods such as ANOVA. The study only allows us to detect the effect size of different sample grouping schemes without the control of confounding factors such as age, occupation, and other personal behavioral factors.

\section{Conclusions}

Our study showed that women without reproductive tract infections or in pregnancy had decreased vaginal microbial richness and beta diversity. The compositional ratio of the main lactobacillus species may shift depending on the normal physiological cycle and reproductive tract infections. We found no bacterial vaginosis associated bacteria in addition to Gardnerella. The species level profile of Lactobacillus were also differed from other studies. We suspect this discrepancy was due to the different source of samples on whom there were diverse formulae of antibiotic administration, for instance, antimicrobials abuse is popular in present in China.

\section{Methods}

\section{Study subjects}

111 women at reproductive age (32.61 \pm 7.75 years old) were recruited at Qilu Hospital of Shandong University and the 5th People's Hospital of Jinan city, China, and were grouped by reproductive tract infections (gynecological diagnosis), childbearing history and the phase of menstrual cycle on the day the specimen swabs were collected(Additional file: Figure S1).

Generally, the first day of menstruation to the day before the next menstruation is called a menstrual cycle [27]. According to the average 28 days of each menstrual cycle, 111 women of childbearing age in this study were divided into 3 stages in proportion to the average menstrual cycle:

(1) Follicular_phase is equivalent to the interval from the 5th to 14th day of a menstrual cycle.

(2) Luteal_phase is the period from the beginning of ovulation to the next menstruation, i.e. the 15th to 28th day of a menstrual cycle.

(3) In_pregnancy was defined as $\geq 40$ days pregnancy and before parturition. Because women in menstrual period (equivalent to the period from the 1st to 4th day of a menstrual cycle) did not visit the hospital, there were no women of menstrual period recruited to this study.

The grouping of childbearing history was performed with respect to the results of questionnaire and clinical examination.

Nugent score and Amsel's criteria in combination with clinical symptoms were used to identify normal women from groups of different reproductive tract infections[28]. 
Individuals with antibiotic usage within the last 2 weeks or those with examination and treatment of urogenital tract within the last 3 days were excluded. The study protocol was approved by the study review board and ethic committee of Institute of Basic Medicine囚Shandong First Medical University \& Shandong Academy of Medical Sciences. All the subjects signed informed consent and filled out questionnaires.

\section{Biospecimen collection}

We got two swabs from each subject with one dry swab thoroughly wiped the lateral and posterior fornix of the vaginal wall to collect the complete population of microbiome, while the other physiological saline soaked swab were used to extract vaginal discharge for measurement of $\mathrm{pH}$ and direct detection of mycotic infection under optical microscopy.

\section{S rRNA gene sequence analysis}

The DNA was extracted from the dry swab of each sample as described previously[25,29]. The V3-V4 regions of the 16S rRNA gene were amplified using PCR and sequenced on the Illumina MiSeq platform with the Illumina RTA software for image recognition and basecalling, and Illumina bcl2fastq 2.17 software for demultiplexing at Suzhou GENEWIZ ${ }^{R}$ Biotechnology Co., Ltd, China[30].

A total of 7,041,153 high-quality(Q30(\%): 71.78-95.03) sequences was obtained with a median read count per sample of 59,560 (range: 40,173-174,385)(Additional file:Table S6). These single-end reads were processed for quality filtering, denoising, and chimera removal to form Amplicon Sequence Variants(ASVs, also known as Operational Taxonomy Units(OTUs)) using the DADA2 plugin[31] in QIIME2(Quantitative Insights into Microbial Ecology, version: 2019.10 (1572640561))[32]. OTUs with only one read were excluded.

Alpha diversity was estimated by the measure of observed_otus, Shannon's Index(using information of OTU frequency)[33], chao1 and PD_whole_tree(using information of phylogenetic relationship of different OTUs)[34] using a sampling depth range of 10-25,000 seqences/sample to generate 10 rarefied OTU tables and compute alpha diversity metrics for each rarefied OTU table. Beta diversity was measured by unweighted UniFrac, weighted UniFrac and bray_curtis distance based on the 25,000 seqences/sample rarefied OTU table[35]. Relative abundances of taxa summarized at the L2(Phylum) to L6(genus) levels were also respectively calculated from the 25,000 seqences/sample rarefied OTU table. These analyses were conducted using the work flow core_diversity_analyses.py in QIIME(version 1.9.1)[36] and Green genes data based classifier (version gg-13-8-99-nb)[37] as the taxonomic reference.

\section{Statistical analysis}

The tests of alpha diversity(PD_whole_tree, chao1, observed_otus and Shannon's Index) and beta diversity (unweighted UniFrac, weighted UniFrac and bray_curtis distance) differences between groups were performed using a two-sided Student's two-sample t-test embedded in the QIIME 1.9.1 work flow 
core_diversity_analyses.py. Taxa relative abundance differences over groups of gynecological diagnosis, childbearing history and menstrual cycle were estimated based on parametric ANOVA and nonparametric kruskal_wallis in QIIME 1.9.1 command group_significance.py. The significant relative abundance differences of 3 bacterial genera were intensively compared between each 2 groups by Post Hoc Bonferroni multiple comparisons in SPSS(version 22.0). Bonferroni correction was used to adjust for tests of multiple taxa. $P$ values less than 0.05 were considered significant after adjustment for multiple tests.

\section{Abbreviations}

ANOVA: analysis of variance; ASVs: amplicon sequence variants; BV: bacterial vaginosis; BVAB: bacterial vaginosis associated bacteria; IQR: interquartile range; MV: mycotic vaginitis; NUC: nongonococcal urethritis/cervicitis; OTU: operational taxonomical unit; PCoA: principal co-ordinates analysis; PD_whole_tree: phylogenetic diversity; QIIME: quantitative insights into microbial ecology

\section{Declarations}

\section{Funding}

This work was supported by the Academic Promotion Programme of Shandong First Medical University(2019QL007).

\section{Authors' contributions}

Y.T.D and H.Y.L designed the study. L.J.C, Q,X and X.Z.X collected the samples. Y.G, Y.R.Land H.W performed the sequencing. Y.T.D and $Y G$ analyzed the data and drafted the manuscript. H.Y.S supervised the statistical analysis. Y.T.D, Y.G, H.Y.L and Q.X did the data illustration and graphical display. Y.T.D and H.Y.L did the manuscript revision. All authors read and approved the final manuscript.

\section{Competing interests}

The authors declare that they have no competing interests.

\section{Consent for publication}

The consent forms signed by each participant included their consent to allow us to publish our findings.

\section{Ethics approval and consent to participate}

This study was approved by the study review board and ethic committee of Institute of Basic Medicine区 Shandong First Medical University \& Shandong Academy of Medical Sciences. All participants signed informed consent to participate in the study.

\section{Author details}


${ }^{1}$ Zhangdian district Center for Disease Control and Prevention, Zibo,255000, China; ${ }^{2}$ The Second Affiliated Hospital, Shandong University of Traditional Chinese Medicine, Jinan, 250001, China; ${ }^{3}$ Qilu Hospital of Shandong University, Jinan, 250012, China; ${ }^{4}$ Obstetrics and Gynecology Department, The $5^{\text {th }}$ People's Hospital of Jinan, Jinan,250022, China; ${ }^{5}$ Institute of Basic Medicine, Shandong First Medical University \& Shandong Academy of Medical Sciences, Jinan, 250062, China;

\section{Reference}

1. Lori Uscher-Pines, Alexandra L. Hanlon, Deborah B. Nelson. Racial Differences in Bacterial Vaginosis among Pregnant Women: The Relationship between Demographic and Behavioral Predictors and Individual BV-Related Microorganism Levels. Matern Child Health J.2009;13:512-519.

2. Jayshree R.S., Kumar R.V. Contribution of the Gut and Vaginal Microbiomes to Gynecological Cancers. In: Mehta S., Singla A. editors. Preventive Oncology for the Gynecologist. Springer, Singapore. 2019;p.399-416.

3. Regan JA, Klebanoff MA, Nugent RP, Eschenbach DA, Blackwelder WC, et al. Colonization with group B streptococci in pregnancy and adverse outcome. VIP Study Group. Am J Obstet Gynecol.1996;174:1354-1360.

4. Krohn MA, Thwin SS, Rabe LK, Brown Z, Hillier SL. Vaginal colonization by Escherichia coli as a risk factor for very low birth weight delivery and other perinatal complications. $J$ Infect Dis. 1997;175:606-610.

5. Hillier SL, Krohn MA, Cassen E, Easterling TR, Rabe LK, et al. The role of bacterial vaginosis and vaginal bacteria in amniotic-fluid infection in women in preterm labor with intact fetal membranes. Clin Infect Dis. 1995;20:S276-278.

6. Group NIHHMPW, Peterson J, Garges S, et al. The NIH Human Microbiome Project. Genome Res.2009;19:2317-2323.

7. Torondel B., Sinha S., Mohanty J.R., et al. Association between unhygienic menstrual management practices and prevalence of lower reproductive tract infections: a hospital-based cross-sectional study in Odisha, India. BMC Infect Dis. 2018;18:1-12.

8. Rebecca M Brotman, Xin He, Pawel Gajer, et al. Association between cigarette smoking and the vaginal microbiota: a pilot study. BMC Infect Dis. 2014;14:1-11.

9. Bonnie Chaban, Matthew GLinks, Teenus Paramel Jayaprakash, et al. Characterization of the vaginal microbiota of healthy Canadian women through the menstrual cycle. Microbiome.2014;2:23.

10. Roberto Romero, Sonia S Hassan, Pawel Gajer, et al. The composition and stability of the vaginal microbiota of normal pregnant women is different from that of non-pregnant women. Microbiome.2014;2:4.

11. Yulian Chen, Zubei Hong,Wenjing Wang, et al. Association between the vaginal microbiome and highrisk human papillomavirus infection in pregnant Chinese women. BMC Infectious Diseases.2019;19:677. 
12. Haohui Zhong, Laura Lehtovirta-Morley, Jiwen Liu, et al. Novel insights into the Thaumarchaeota in the deepest oceans: their metabolism and potential adaptation mechanisms. Microbiome. 2020; 8:78.

13. Ohana Y. A. Costa, Mattias de Hollander, Agata Pijl, et al. Cultivation-independent and cultivationdependent metagenomes reveal genetic and enzymatic potential of microbial community involved in the degradation of a complex microbial polymer. Microbiome. 2020;8:76.

14. Jing Wu, Brandilyn A Peters, Christine Dominianni, et al. Cigarette smoking and the oral microbiome in a large study of American adults. ISME J.2016;1-12.

15. Ravel J, Gajer P, Abdo Z, et al. Vaginal microbiome of reproductive-age women. Proc Natl Acad Sci.2011;108(Suppl1):4680-4687.

16. Gajer P, Brotman RM, Bai G, et al. Temporal dynamics of the human vaginal microbiota. Sci Transl Med. 2012;4(132):132 ra152.

17. Hickey RJ, Abdo Z, Zhou X, et al. Effects of tampons and menses on the composition and diversity of vaginal microbial communities over time. BJOG. 2013;120:695-704.

18. Verstraelen $H$,Verhelst $R$,Claeys $G$, et al.Longitudinal analysis of the vaginal microflora in pregnancy suggests that L.crispatus promotes the stability of the normal vaginal microflora and that L.gasseri and/or L.iners are more conducive to the occurrence of abnormal vaginal microflora. BMC Microbiol.2009;9:116.

19. Witkin SS, Mendes-Soares H, Linhares IM, et al. Influence of vaginal bacteria and D- and L- lactic acid isomers on vaginal Extracellular matrix metalloproteinase inducer: implications for protection against upper genital tract infections. mBio.2013;4:e00460-13.

20. Fredricks DN. Molecular methods to describe the spectrum and dynamics of the vaginal microbiota. Anaerobe.2011;17:191-195.

21. Mitchell C, Manhart LE, Thomas K, et al. Behavioral predictors of colonization with Lactobacillus crispatus or Lactobacillus jensenii after treatment for bacterial vaginosis: a cohort study. Infect Dis Obstet Gynecol.2012;2012:706540.

22. Ravel J, Brotman RM, Gajer P, et al. Daily temporal dynamics of vaginal microbiota before, during and after episodes of bacterial vaginosis. Microbiome.2013; 1:29.

23. Kaewsrichan J, Peeyananjarassri K, Kongprasertkit J. Selection and identification of anaerobic lactobacilli producing inhibitory compounds against vaginal pathogens. FEMS Immunol Med Microbiol. 2006;48:75-83.

24. Kaewsrichan J, Peeyananjarassri K, Kongprasertkit J. Selection and identification of anaerobic lactobacilli producing inhibitory compounds against vaginal pathogens. FEMS Immunol Med Microbiol.2006;48:75-83.

25. Qing Xia, Lijuan Cheng, Hua Zhang, et al. Identication of vaginal bacteria diversity and it's association with clinically diagnosed bacterial vaginosis by denaturing gradient gel electrophoresis and correspondence analysis. Infection, Genetics and Evolution.2016;44:479-486. 
26. Ma B, Brotman RM, Gajer P, et al. Association between Chlamydia trachomatis Genital infections and the vaginal microbiome. In The International Society for Sexually Transmitted Disease Research, $20^{\text {th }}$ Biennial Congress: Vienna, Austria, July14-17,2013.

27. Karl Martin Pirke,Wolfgang Wuttke,Ulrich Schweiger. The Menstrual Cycle and Its Disorders. Springer, Berlin, Heidelberg,1989.ISBN(online) : 978-3-642-74631-4.

28. Nugent RP, Krohn MA, Hillier SL. Reliability of diagnosing bacterial vaginosis is improved by a standardized method of gram stain interpretation. Journal of Clinical Microbiology.1991;29:297.

29. Yutao Diao, Xueqiang Fang, Qing Xia, et al. Organism diversity between women with and without bacterial vaginosis as determined by polymerase chain reaction denaturing gradient gel electrophoresis and 16S rRNA gene sequence. Obstetrics and Gynaecology Research.2011; 37:14381446.

30. https://www.genewiz.com/en/Public/Services/Next-Generation-Sequencing

31. Callahan B J, McMurdie $P J$, Rosen M J, et al. DADA2: High-resolution sample inference from Illumina amplicon data. Nature Methods. 2016;13:581-583.

32. Evan Bolyen, Jai Ram Rideout, Matthew R Dillon, et al.

QIIME 2: Reproducible, interactive, scalable, and extensible microbiome data science. Peer J Preprints.2018. https://doi.org/10.7287/peerj.preprints.27295v2.

33. Shannon CE. The mathematical theory of communication. MD Comput.1997;14:306-317.

34. Faith DP, Baker AM. Phylogenetic diversity (PD) and biodiversity conservation: some bioinformatics challenges. Evol Bioinforma. 2006;2:121-128.

35. Lozupone C, Lladser ME, Knights D, et al. UniFrac: an Effective distance metric for microbial community comparison. ISME J. 2011;5:169-172.

36. Caporaso, J. G., et al. QIIME allows analysis of high-throughput community sequencing data. Nat Methods. 2010;7: 335-336.

37. http://data.qiime2.org/2019.10/common/gg-13-8-99-nb-classifier.qza.

\section{Figures}




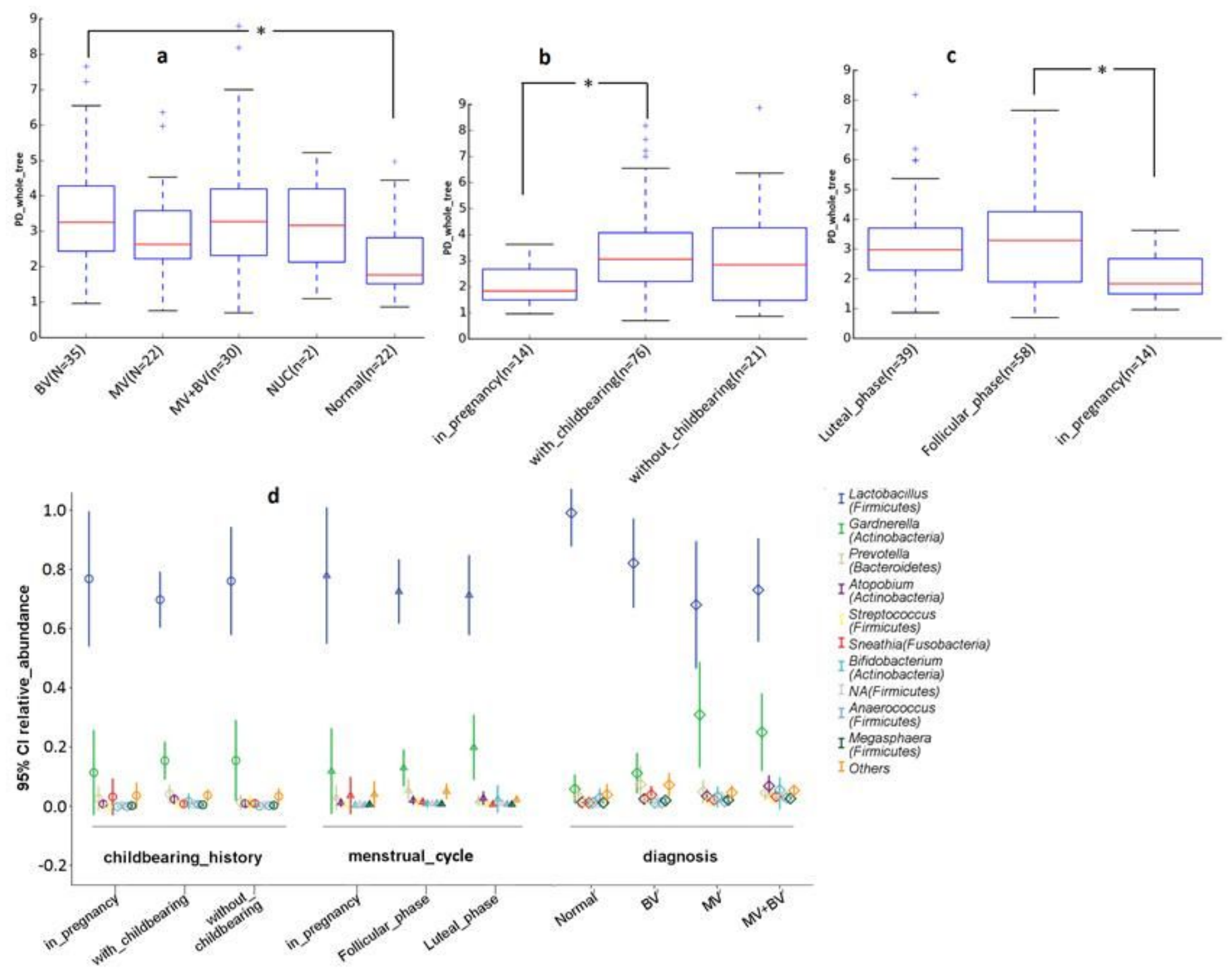

Figure 1

a. Alpha diversity by PD_whole_tree was lower in Normal women than in the other groups of gynecological infections and was significantly lower in Normal women than in women affected BV. b. Women in pregnancy ( $\geq 40$ days gestation and before parturition) had significant lower PD_whole_tree than those with childbearing. c. Women in pregnancy had significant lower PD_whole_tree than those in follicular phase. d. Mean and $95 \%$ interval of genus-relative abundance. There was no genus significantly different in relative abundance by childbearing history and menstrual cycle according to both the ANOVA and nonparametric kruskal_wallis with Bonferroni correction. But genus-relative abundances of Gardnerella, Streptococcus, and order-relative abundance of Lactobacillales(NA(Firmcutes)) were significantly different among the 5 groups of gynecological infections. In Figure $1: a, b$ and $c$, the boxes are interquartile range (IQR); median values are the bands within the boxes; the line terminals outside the boxes are upper and lower endpoint of the data; crosses are outliers. In Figure 1: $d$, the lines are 95\% intervals of genus relative abundance; mean values are icons in the middle of the $95 \%$ interval lines. 

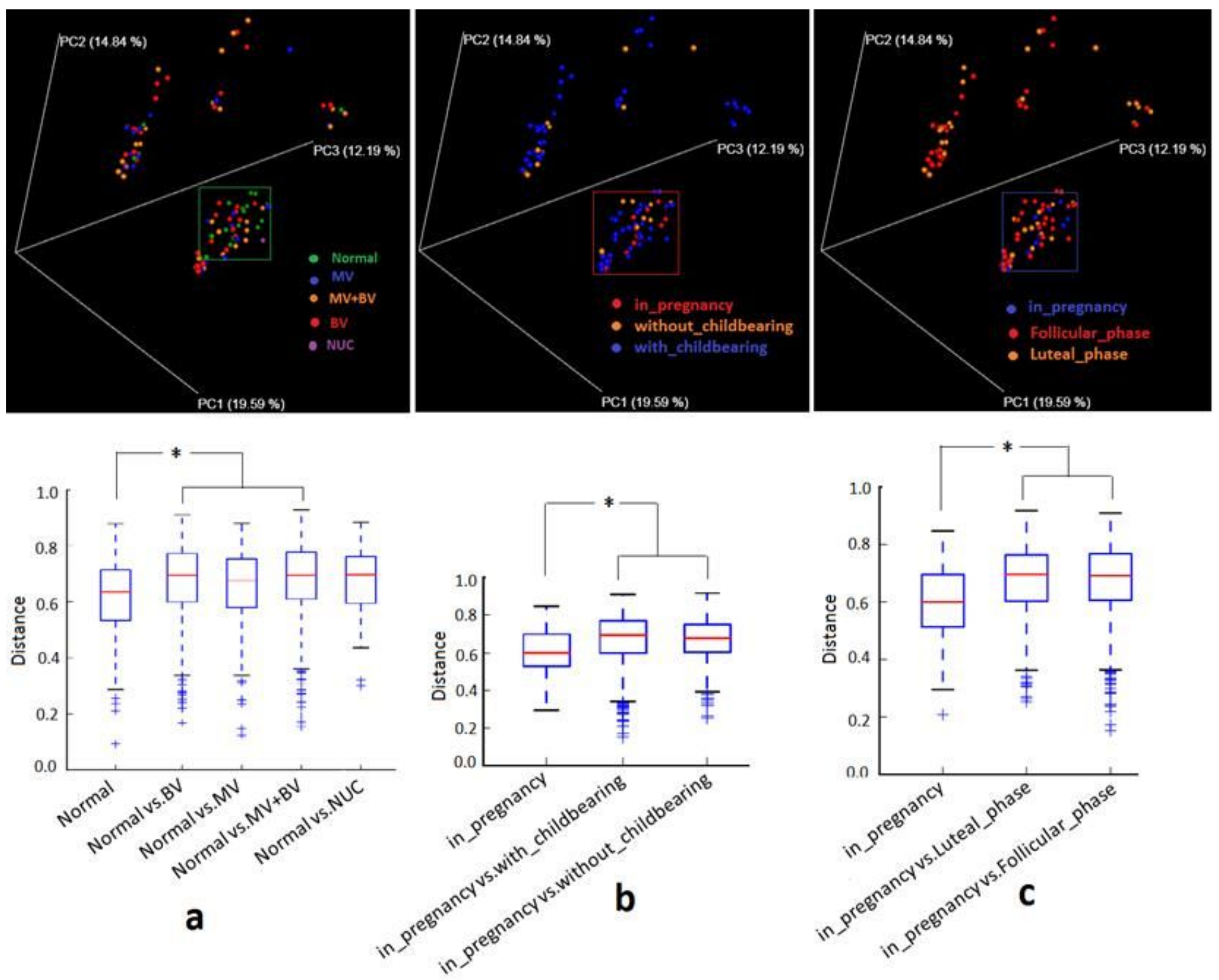

\section{Figure 2}

The permutation of the 111 samples in a 3-dimensional space constructed by principal co-ordinates analysis(PCoA囚using Unweighted UniFrac distance. a upper: Samples in Normal group were clustered together (green dots in the rectangle) and had significant shorter genetic distance apart from samples in the other groups of gynecological infections. a lower: Unweighted UniFrac distance (beta diversity) of within-group (Normal) was significantly shorter versus that of between-groups (Normal vs the other gynecological infections). b upper: samples from in pregnancy group were clustered together (red dots in the rectangle) apart from samples in groups of with and without childbearing. b lower: Unweighted UniFrac distance of within-group (in pregnancy) was significantly shorter versus that of between-groups (in pregnancy vs with or without childbearing). c upper: samples from in pregnancy group were clustered together (blue dots in the rectangle) and had significant genetic distance from samples in groups of luteal and follicular phase. c lower: Unweighted UniFrac distance of within-group (in pregnancy) was significantly shorter versus that of between-groups (in pregnancy vs in luteal or follicular phase). In Figure 
$2 \mathrm{a}, \mathrm{b}$ and c lower, the boxes are interquartile range (IQR) of Unweighted UniFrac distance; median values of distance are the bands within the boxes; the line terminals outside the boxes are upper and lower endpoint of the data; crosses are outliers. The tests of significance were performed using a two-sided Student's two-sample t-test. P-values were calculated using 999 Monte Carlo permutations.

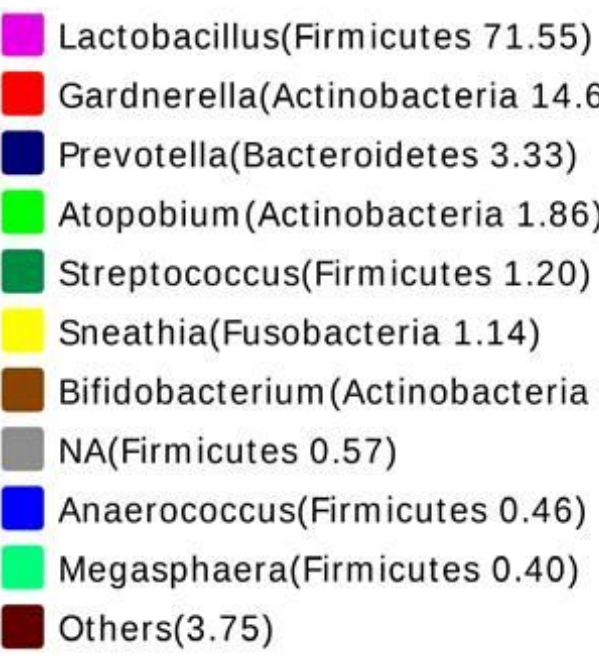

Lactobacillus(Firmicutes 71.55)

Gardnerella(Actinobacteria 14.66)

Atopobium (Actinobacteria 1.86)

Streptococcus(Firmicutes 1.20)

Sneathia(Fusobacteria 1.14)

Bifidobacterium (Actinobacteria 1.07)
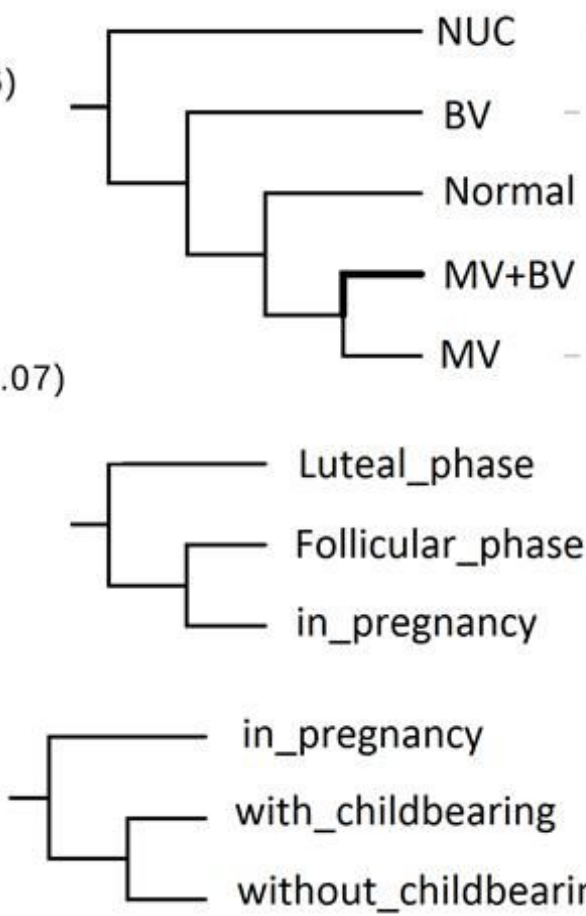

in_pregnancy

with_childbearing

without_childbearing

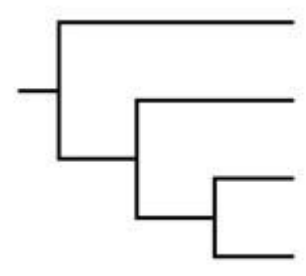

no_pregnancy+ infections

no_pregnancy+Normal

in_pregnancy+Normal

in_pregnancy+infections
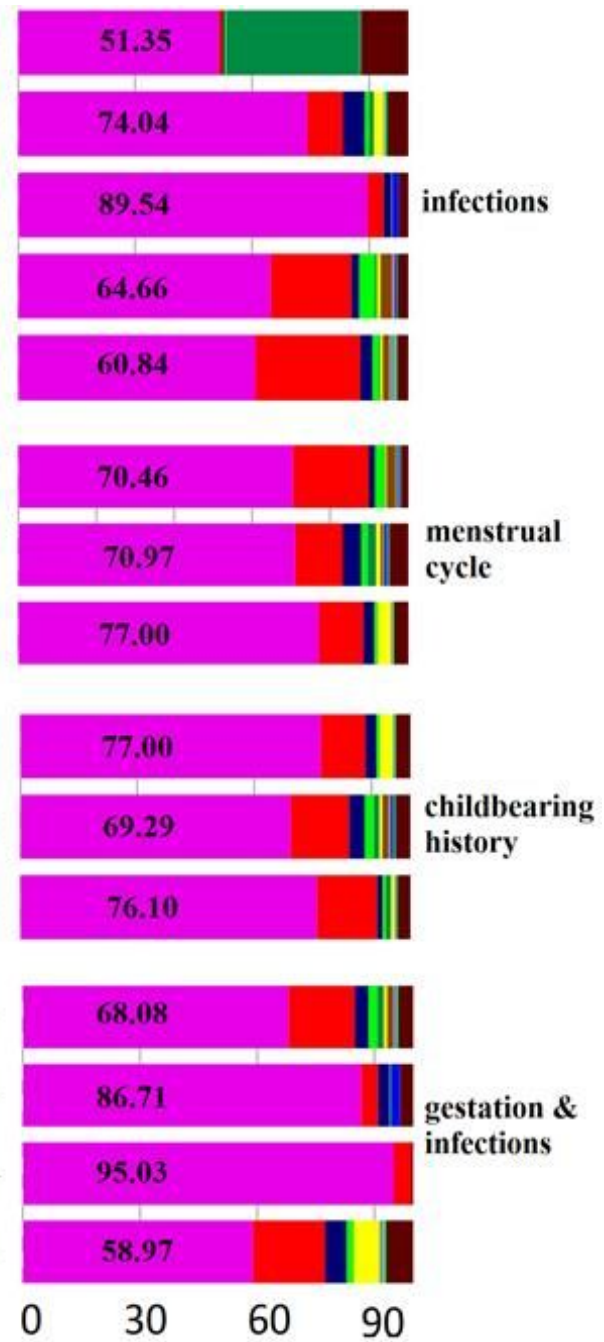

\section{Figure 3}

The compositional profiles of the top 10 genera and dendrogram of the 4 sample grouping schemes(participants were grouped based on infections, menstrual cycle, childbearing history and infections incorporation with gestation, respectively). The left side shows the genera and phylum that the genus is affiliated to, with the average relative abundance(\%) in the total 111 women is shown in parenthesis after each genus. The dendrogram in the middle was based on hierarchical clustering using complete linkage of Unweighted UniFrac distance of the OUT table. Each horizontal bar in the right represents the compositional profile averaged within each sample group wherein the number in each bar indicates the percentage of genus lactobacillus. Only the top 10 most abundant genera are shown. 


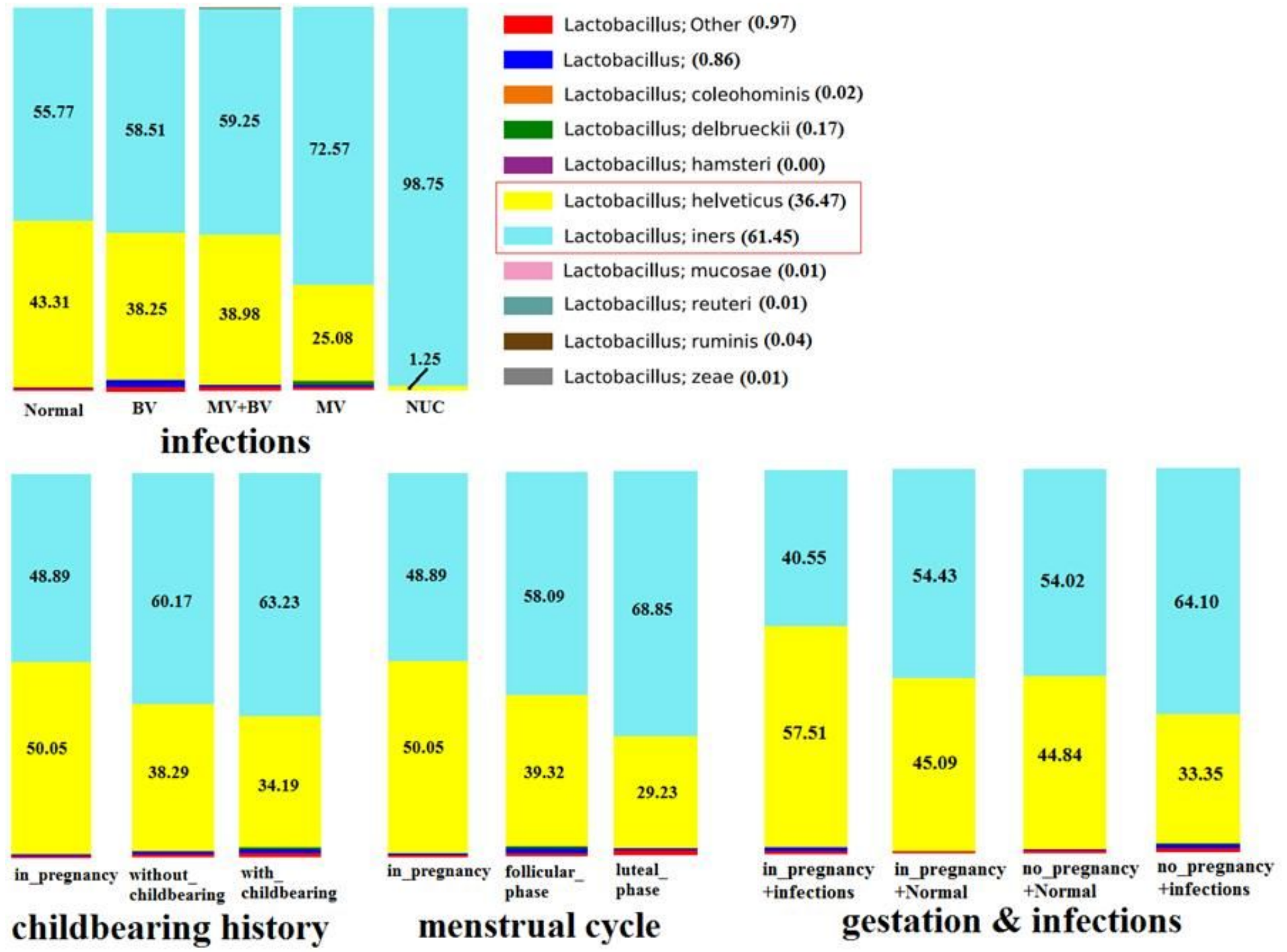

Figure 4

The compositional profiles of the 11 lactobacillus species in 4 sample grouping schemes(participants were grouped based on infections, menstrual cycle, childbearing history and infections incorporation with gestation, respectively). The average relative abundance(\%) in the total 111 women is shown in parenthesis after each species. Each bar represents the compositional profile of lactobacillus species averaged within each sample group with the average relative abundance(\%) only of the 2 most abundant species(lactobacillus iners and lactobacillus helveticus) are shown.

\section{Supplementary Files}

This is a list of supplementary files associated with this preprint. Click to download.

- SupplementaryMaterial.docx 